Williams Smith, H. (1951). J. gen. Microbiol. 5, 472-479.

\title{
The Typing of Salmonella thompson by Means of Bacteriophage
}

\author{
By H. WILLIAMS SMITH \\ Animal Health Trust, Livestock Research Station, \\ Houghton Grange, Huntingdon
}

\begin{abstract}
SUMMARY: By the use of seven phage preparations, forty-one of fifty smooth strains of Salmonella thompson were classified into eleven types. The remaining nine strains were insusceptible to all the test phages; mouse passage did not render them susceptible. Acquired phage resistance was responsible for many of the strains being regarded as different phage types. A study of phage-resistant variants cast doubt on the complete validity of Bail's cross-resistance tests. The danger of using lysogenic cultures for the propagation of phages for the study of phage mutation were noted. Most of the $S$. thompson phages were fully active against strains of $S$. cholerae-suis but not for other Salmonella sp. that were tested.
\end{abstract}

In a recent study (Williams Smith, 1951) a number of bacteriophages were isolated from lysogenic strains of $S$. thompson. The purpose of this present paper is to report observations that were made while evolving a method of typing strains of $S$. thompson by the use of these phages.

\section{MATERIALS AND METHODS}

The method of isolation, purification, and propagation of the phages used in this study, and the method of titration of the final phage preparations, the preparation of phage-resistant strains and the differentiation of the phages from each other by cross-resistance tests have been reported elsewhere(Williams Smith, 1951).

Method of setting-up tests. A modification of the method devised by Wilson \& Atkinson (1945) for the phage typing of staphylococci was used. Nutrient agar plates containing $2 \%$ agar were dried at $37^{\circ}$ for $2 \mathrm{hr}$. with the lids partly raised. Four drops $(0.08 \mathrm{ml}$.) of an $18 \mathrm{hr}$. broth culture of the strain to be typed were spread evenly over the surface of a plate by means of a glass spreader. After the plates had dried, a standard loopful of each phage preparation in test dilution, i.e. the highest dilution capable of producing confluent lysis of its propagating strain, was spotted on to the plate. When several strains were tested, the phage preparations were spotted on to each plate by means of a dropping pipette (150 drops $/ \mathrm{ml}$.). For the sake of convenience, the bottom of each plate was marked into a number of squares equal to the number of phage preparations in use, by means of a grease pencil. When the drops had been absorbed, the plates were incubated at $37^{\circ}$ overnight, and read next morning.

All the phage preparations were purified and propagated on strain 19, which, as far as could be determined, was non-lysogenic and did not show any evidence of acquired phage resistance. Each phage was designated by the number of the lysogenic strain from which it was isolated, followed by the number of the 
strain upon which it was propagated, i.e. strain 19 . One of the phage preparations, 6/19, was used in two dilutions, one 100 times stronger than the normal test dilution, as it was found that the stronger preparation was capable of lysing some strains which would otherwise be considered untypable. The strong preparation for convenience was designated 6/12/19 simply because in this strength it has been found capable of lysing strain 12, a strain previously regarded as untypable.

\section{RESULTS}

The results of the phage typing of fifty smooth strains of $S$. thompson by means of seven phage preparations are shown in Table 1. Of the fifty strains, fortyone $(82 \%)$ were typable and nine $(18 \%)$ were not acted upon by any of the

Table 1. The phage typing of fifty strains of Salmonella thompson

\begin{tabular}{|c|c|c|c|c|c|c|c|c|c|}
\hline \multirow{2}{*}{$\begin{array}{l}\text { Phage } \\
\text { type }\end{array}$} & \multicolumn{7}{|c|}{ Phage preparations } & \multicolumn{2}{|c|}{$\begin{array}{l}\text { Strains of } \\
\text { S. thompson }\end{array}$} \\
\hline & $7 / 19$ & $5 / 19$ & $8 / 19$ & $13 / 19$ & $2 / 19$ & $6 / 19$ & $6 / 12 / 9$ & No. & $\%$ \\
\hline 1 & CL & CL & CL & CL & CL & CL & CL. & 2 & 4 \\
\hline 2 & - & CL & CL & CL & CL & CL & CL & 1 & 2 \\
\hline 3 & - & - & $\mathbf{C L}$ & CL & CL & CL & CL & 8 & 16 \\
\hline 4 & - & CL & - & CL & $\mathbf{C L}$ & CL & CL & 1 & 2 \\
\hline 5 & - & - & - & CL & CL & CL & CL & 1 & 2 \\
\hline 6 & - & CL & + & - & CL & CL & CL & 3 & 6 \\
\hline 7 & CL & - & CL & - & CL & + & CL & 2 & 4 \\
\hline 8 & CL & - & - & + & - & CL & CL & 1 & 2 \\
\hline 9 & - & - & - & - & - & CL & CL & 14 & 28 \\
\hline 10 & - & - & - & - & - & - & CL & 6 & 12 \\
\hline 11 & CL & - & + & - & + & - & ++ & 2 & 4 \\
\hline Untypable & - & - & - & - & - & - & - & 9 & 18 \\
\hline
\end{tabular}

Confluent lysis $=\mathrm{CL}$; semi-confluent plaques $=++$; discrete plaques $=+$; no lysis $=-$.

The phage preparations were used in the test dilution with the exception of phage 6/12/19, which was used in a strength capable of producing confluent lysis of an hitherto untypable strain, strain 12. The phage types have been numbered only for the purpose of this table and are not recognized designations.

phages. The forty-one typable strains could be divided into eleven types. Apart from one type, type 9 , which comprised $28 \%$ of the whole, the number of other types were not large. The fifty strains were retested several times during a period of 6 months and, apart from the variations inherent in any method of phage-typing which depends on the pattern reactions given by strains with a number of test phages, they showed no significant variation in their susceptibility. During this period they were subcultured a number of times. Thus, judging only from strains maintained under laboratory conditions, the different phage types appeared to be relatively stable.

Twenty of the fifty strains were responsible for different outbreaks of food poisoning in human beings, and thirty were isolated from domestic animals. Phage typing did not serve to differentiate the animal strains from the human strains. No real opportunities have presented themselves to determine whether this method of typing would be of any value in studying the epidemiology of $S$. thompson infections. Three horses belonging to the same stud were found to 
be excreting the same phage type (type 9) of $S$. thompson, and three hens all from the same flock were also infected with an identical phage type (type 10). The faeces from a horse were examined several times over a period of 6 months and found to contain the same phage type (type 5) of $S$. thompson on each occasion.

\section{The significance of lysogenic strains in phage typing}

In a previous communication (Williams Smith, 1948) it was shown that acquired phage-resistance was responsible for many lysogenic strains of staphylococci being classified as different phage types. Table 1 shows that two strains (type 1 ) of $S$. thompson exhibited no evidence of acquired phageresistance as they were fully susceptible to all the test phages. They were nonlysogenic. The remaining forty-eight strains showed some degree of resistance to one or more of the phages, and it was possible to show by repeated testing that forty-two of them were lysogenic. It was, therefore, considered possible that the phage resistance they had acquired, as indicated by the fact that they were lysogenic, may have been responsible for their resistance to some of the test phages. It was decided to investigate this possibility. The investigations were carried out in a manner similar to that which had been successful in elucidating the relationship among the staphylococcal strains. The principle of the method was to prepare a resistant variant of a strain (in this case a type 1 strain, strain 19), showing no evidence of acquired phage-resistance, to the phage or phages carried by a lysogenic strain that was resistant to one or more of the test phages. The resistant variant and the lysogenic strain were then tested with the test phages, and if they reacted in exactly the same manner it was concluded that acquired phage resistance was responsible for the lysogenic strain being considered to be a different phage type to type 1 . The technical details of the method were as follows. A young broth culture of the lysogenic strain was maintained at $56^{\circ}$ for $30 \mathrm{~min}$. and then tested for bacterial sterility. Resistant variants of strain 19 were prepared to the 'crude phage' contained in the heat-treated culture. 'Crude phage' was used instead of purified phage as some strains were shown to be carrying more than one phage. The results of these experiments are shown in Table 2. The results obtained with the lysogenic strains $1,2,3,14,25,47$ and 288 and the resistant variants of strain 19 to the phages carried by these strains indicated that acquired phage-resistance accounted for these lysogenic strains being regarded as different phage types. Two types of resistant variants of strain 19 to the phage or phages carried by strain 288 were noted. One of them, $19(288) a$, behaved exactly the same as strain 288 and the other, $19(288) b$, was completely resistant to all the test phages. No evidence was obtained to show that acquired phage-resistance accounted for the classification of strains $5,17,49$ and 7 as different phage types, although the resistant variants of strain 19 to the phages carried by these strains demonstrated that the phages they carried could account for the resistance of these strains to some, but not all, of the test phages. It may well be that the resistance exhibited by these strains had been acquired following infection with more than one phage in the field, and that they were now carrying only one phage; it had been shown (Williams Smith, 1951) that after strains 
Table 2. The action of the test phages on lysogenic strains and on variants of strain 19 resistant to the phages carried by these lysogenic strains

\begin{tabular}{lcllllll}
\multicolumn{7}{c}{ Phage preparations } \\
Strain & $7 / 19$ & $5 / 19$ & $8 / 19$ & $13 / 19$ & $2 / 19$ & $6 / 19$ & $6 / 12 / 19$ \\
19 & CL & CL & CL & CL & CL & CL & CL \\
1 & CL & - & CL & - & CL & + & CL \\
$19(1)$ & CL & - & CL & - & CL & + & CL \\
2 & - & - & - & - & - & CL & CL \\
$19(2)$ & - & - & - & + & - & CL & CL \\
14 & - & - & - & - & - & CL & CL \\
$19(14)$ & - & - & - & - & - & CL & CL \\
3 & - & - & CL & CL & CL & CL & CL \\
$19(3)$ & - & - & CL & CL & CL & CL & CL \\
25 & - & - & CL & CL & CL & CL & CL \\
$19(25)$ & - & - & CL & CL & CL & CL & CL \\
47 & CL & - & + & - & - & - & - \\
$19(47)$ & CL & - & + & - & - & - & - \\
288 & - & - & - & CL & CL & CL & CL \\
$19(288) a$ & - & - & - & CL & CL & CL & CL \\
$19(288) b$ & - & - & - & - & - & - & - \\
5 & - & - & - & - & - & - & - \\
$19(5)$ & + & - & - & - & - & CL & CL \\
17 & - & - & - & - & - & CL & CL \\
$19(17)$ & CL & - & CL & CL & CL & CL & CL \\
49 & CL & - & - & + & - & CL & CL \\
$19(49)$ & CL & CL & CL & - & CL & CL & CL \\
7 & - & - & - & - & - & - & CL \\
$19(17)$ & - & - & CL & CL & CL & CL & CL \\
8 & - & CL & - & CL & CL & CL & CL \\
$19(8)$ & - & - & - & - & - & - & + \\
19 & & - & & - & & &
\end{tabular}

The resistant variants of strain 19 are designated by the number 19 followed by the number, in brackets, of the lysogenic strain from which the 'crude' phage was obtained. All the phages, except 6/12/19, were used in test dilution.

Symbols as in Table 1 .

of $S$.thompson were made resistant in the laboratory to two phages they frequently only carried one of them, the one to which they had first been made resistant. Alternatively, other phenomena may be responsible for the phage reactions of these four strains. The reactions of strain $19(8)$, the variant of strain 19 made resistant to the phage carried by strain 8 , were difficult to explain, since this variant was resistant to seven test phages, whereas strain 8 was only resistant to two of them. This resistant variant resembled strain $19(288) b$, since the induction of variants by bacteriophage action in both cases gave rise to strains which were resistant to all the test phages. Since both these variants were smooth, this change cannot be attributed to smooth-rough variation; but rather that phage action had induced variants lacking receptors for all phages and not only for the phage that had been used for their induction which, presumably, happens in most cases. These results question the validity of Bail's (1923) cross-resistance tests, since the essential part of these tests is that 
the variants, as far as phage resistance is concerned, should portray accurately the character of the phages which were responsible for their production. It was mentioned earlier that six of the forty-eight strains of $S$. thompson which showed some degree of resistance to the test phages could not be shown to be lysogenic, despite repeated attempts and, therefore, there was no evidence that they had acquired resistance as a result of infection in the field. It is well known that strains may be made resistant without subsequently being latently infected with phage, and this may have occurred in the case of these six strains.

That a lysogenic strain may carry two phages, both of which may have had some influence on the phage susceptibility of the strain was shown in the case of strain 14. The phage reactions of resistant variants of strain 19 to each of the two phages, $A$ and $B$, carried by strain 14 and to both of these phages is shown in Table 3. Both phages were responsible in part for strain 14 being classified as a distinct phage type (type 9 ).

Table 3. The action of the typing phages on variants of strain 19 resistant to the phages carried by strain 14

\begin{tabular}{|c|c|c|c|c|c|c|c|}
\hline \multirow[b]{2}{*}{ Strain } & \multicolumn{7}{|c|}{ Phage preparations } \\
\hline & $7 / 19$ & $5 / 19$ & $8 / 19$ & $13 / 19$ & $2 / 19$ & $6 / 19$ & $6 / 12 / 19$ \\
\hline 19 & CL & CL & CL & CL & CL & CL & CL \\
\hline $19($ resistant to phage $A)$ & - & - & CL & CL & CL & CL & CL \\
\hline 19 (resistant to phage $B$ ) & CL & + & - & - & - & CL & CL \\
\hline $\begin{array}{l}19 \text { (resistant to phages } \\
A \text { and } B \text { ) }\end{array}$ & - & - & - & - & - & CL & CL \\
\hline 14 & - & - & - & - & - & CL & CL \\
\hline
\end{tabular}

Strain 14 was latently infected with two phages, $A$ and $B$.

Symbols as in Table 1 .

\section{The effect of mouse passage on phage susceptibility}

Table 1 shows that nine of the fifty strains of $S$. thompson were untypable. In view of the observation of Atkinson, Woodroofe, Macbeth, Chibnall \& Mander (1949) that phage-resistant strains of $S$. blegdam could be rendered susceptible to the action of phages by mouse passage, six of the nine strains of $S$. thompson that were resistant to all the test phages were subjected to this procedure. They were then retested but were still found to be resistant. Atkinson $e t$ al. also noted that the virulence for mice of phage-susceptible strains of $S$. blegdam appeared greater than that of the resistant strains. No obvious difference was noted in the present work between the virulence of phage-resistant and phage-susceptible strains, although the numbers of mice used were too small to draw any definite conclusion.

\section{The adaptation of phages to lyse untypable strains}

The adaptation of phages to lyse bacteria previously considered untypable, by the selection and propagation of mutants, was first utilized by Craigie \& Yen (1938) in the typing of $S$. typhi, and has since been used by other workers. Apparent mutation of one of the staphylococcal phages of Wilson \& Atkinson 
(1945) was shown by Rountree (1949) to be due to the phage preparation used for adaptation having been previously contaminated by another phage liberated during propagation on a lysogenic strain. This contaminating phage was liberated from the lysogenic strain. It was decided to investigate this phenomenon with regard to the $S$. thompson phages. A phage carried by strain 7 was propagated on the lysogenic strain 6. This phage 7/6 was 'purified' by plating with a culture of strain 6 , picking single plaques and propagating three times. Attempts were now made to adapt $7 / 6$ to lyse strain 14, a strain at one time regarded as untypable. Phage $7 / 6$ was titrated on strains 14,6 and 19 with the following results :

Dilutions of phage $7 / 6$

$\begin{array}{rccccc}\text { Strain } & 0 & 10^{-1} & 10^{-2} & 10^{-3} & 10^{-4} \\ 14 & ++ & + & - & - & - \\ 6 & \text { CL } & \text { CL } & \text { CL } & ++ & + \\ 19 & \text { CL } & \text { CL } & \text { CL } & ++ & +\end{array}$

A single plaque produced by the activity of phage $7 / 6$ on strain 14 was picked, purified, and propagated on strain 14. The resulting phage preparation, $7 / 6 / 14$, was found to be completely inactive on strain 6 , its previous propagating strain. Further work showed that this phage behaved in exactly the same way as the phage carried by strain 6 ; cross-resistance tests showed these two phages to be completely identical. It was also shown that the phage carried by strain 7 after purification and propagation on the non-lysogenic strain 19, was without action on strain 14, and that it was quite impossible to isolate any mutants from this phage which were capable of lysing strain 14 . It was, therefore, obvious that the apparent adaptation of phage $7 / 6$ to lyse strain 14 was no more than the propagation on strain 14 of the phage carried by the lysogenic strain 6 . This suggested that adequate precautions should be taken when studying phage mutation to ensure that phages are never propagated on lysogenic bacteria, since pure phage preparations cannot be obtained by this procedure; they will inevitably be contaminated by the phage carried by the lysogenic propagating strain.

The lytic action of the Salmonella thompson phages on other species of salmonella

The seven $S$. thompson phages, in the strength used for phage typing, were tested for activity on other species of salmonella. The species examined are listed below with the number of strains of each species placed in brackets.

Group A. S. paratyphi A (2).

Group B. S. paratyphi B (2), typhi-murium (4), stanley (1) saint-paul (1), derby (3), california (1), abortus-equi (1), abortus-ovis (2), abortus-bovis (2), brancaster (1).

Group C. S. paratyphi C (2), cholerae-suis (38), cardiff (1), daytona (1), montevideo (4), oranianburg (2), potsdam (10), concord (9), barielly (2), tennessee (2), muenchen (1), manhattan (1), dusseldorf (1). 
Group D. S. typhi (2), enteritidis (2), dublin (3), rostock (2), moscow (1), blegdam (1), pensacola (1), dar-es-salaam (2), gallinarum (3), pullorum (3).

Group E. S. london (1), give (1), anatum (2), meleagridis (1), zanzibar (1). OTher Groups. S. habana (1), worthington (1), cubana (1), kirkee (1), cerro (1), kentucky (1), minnesota (1), inverness (1).

The phages were completely inactive on all these strains except the one strain of $S$. cardiff and the $S$. cholerae-suis strains. The $S$. cardiff strain was susceptible to phage $6 / 12 / 19$ only, this phage in reality being phage 6/19 used at a higher concentration than that necessary to produce confluent lysis of strain 19. Of the thirty-eight strains of $S$. cholerae-suis, thirty-two were smooth and six rough; sixteen were of the American and twenty-two of the kunzendorf type. Twenty-nine of the thirty-two smooth strains were susceptible to phages $8 / 19$, 2/19,6/19 and 6/12/19; one strain, C15, was also susceptible to phage 7/19. Two smooth strains and the six rough strains were resistant to all the phages. By using $\mathrm{C} 15$ as an indicator strain, twenty-three (88\%) of the twenty-six smooth strains and five $(83 \%)$ of the six rough strains were shown to be latently infected with the same phage, this phage being identical with phage $7 / 19$ (strain $\mathrm{C} 15$ was non-lysogenic). It is probable that this fact would make a satisfactory classification of $S$. cholerae-suis difficult, since most of the strains would exhibit the same degree of phage resistance unless, of course, phages similar to those used by Craigie \& Yen (1938) for typing $S$. typhi could be found.

\section{DISCUSSION}

A position appears to exist in $S$. thompson similar to that in staphylococci (Williams Smith, 1948). That is, acquired phage-resistance was responsible for many strains being classified as different phage types. Under laboratory conditions the phage types appeared to be stable in character. However, if a strain became infected with phage in the field it is probable that an alteration in its susceptibility to the test phages would result, and then the strain would be considered to be a different phage type. That such changes occurred in the field was shown in the case of staphylococci. It is obvious, too, that all lysogenic strains must have undergone at some time a change in phage susceptibility. The primary isolation of salmonella that are actively infected with phage is not a rare occurrence, and it is probable that these changes of type are not unusually rare. Faeces, too, are the natural habitat of salmonella and are a prolific source of different phages. It seems probable therefore that this method of phage typing could be usefully employed during a short-term investigation, such as that of an outbreak of food-poisoning due to $S$. thompson, but that the possibility of change of type may limit the usefulness of this method in investigations conducted over a long period of time. It has to be borne in mind that this method of typing probably classifies together strains with a similar history of phage infection and it would, therefore, be unprofitable to attempt to correlate this classification by phage with other phenomena such as virulence, species of animal host, or clinical symptoms. 


\section{REFERENCES}

Atkinson, N., Woodroofe, G. M., Macbeth, A. M., Chibnall, H. \& Mander, S. (1949). The occurrence of salmonella types in Australia. 5. Salmonella blegdam. Aust. J. exp. Biol. med. Sci. 27, 597.

BaIL, O. (1923). Versuche über die Vielheit von Bakteriophagen. Z. ImmunForsch. $38,57$.

Craigie, J. \& Yen, C. H. (1938). The demonstration of types of B. typhosus by means of preparations of type II phage. Canad. publ. Hlth J. 29, 448, 484.

Rountree, P. M. (1949). The serological differentiation of staphylococcal bacteriophages. J. gen. Microbiol. 3, 166.

Wrlliams Smith, H. (1948). Investigations in the typing of staphylococci by means of bacteriophage. I. The origin and nature of lysogenic strains. II. The significance of lysogenic strains in staphylococcal type designation. J. Hyg., Camb., 46, 74, 82.

Williams Smith, H. (1951). Some observations on lysogenic strains of salmonella. J. gen. Microbiol. 5, 458.

Wrlson, G. S. \& Atrinson, J. D. (1945). The typing of staphylococci by the bacteriophage method. Lancet, i, 647 .

(Received 28 September 1950) 